\title{
Young children can add and subtract by predicting and checking
}

\author{
Osnat Zur, Rochel Gelman* \\ ${ }^{a}$ UCLA, Department of Psychology, University of California, 1282A Franz Hall, Box 951563, Los Angeles, CA, USA \\ ${ }^{\mathrm{b}}$ Center for Cognitive Science and Psychology, Rutgers University, 152 Frellinghuysen Road, Piscataway, NJ 08854, USA
}

\begin{abstract}
We argue that to test preschoolers' understanding of counting, one has to use tasks that relate counting to the goal of doing arithmetic, as counting and arithmetic principles are mutually constrained. A naturalistic study in the preschool classroom led to the development of an "arithmetic-counting" task, where counting was being related to the goal of doing arithmetic. In solving an arithmetic problem, the child viewed the addition or subtraction of $\mathrm{X}$ items from a set with a known number, predicted the answer, and then counted the items in the resultant array to check the prediction. Three experiments, one with 4-year-olds who had relevant in-class experience with the task (Experiment 1), and two others with 4-year-olds (Experiment 2) and 3-year-olds (Experiment 3) who were novices with regard to the task, are reported. All children, even the 3-year-olds, offered reasonable cardinal values during the prediction phase and used counting to check their predictions. Predictions were reliable, in the correct direction or correct, and checking counts were very accurate. Young children's ability to make reasonable predictions and coordinate these with counts "to check" indicated the implicit understanding of counting and arithmetic principles. The theoretical and practical implications of our findings are discussed.
\end{abstract}

(C) 2004 Published by Elsevier Inc.

Keywords: Preschooler; Counting; Predicting

\section{Introduction}

Much of the empirical research on children's early understanding of counting focuses exclusively on counting as a mean to simply determine the cardinality of any set (How many $\mathrm{X}$ are here?), and less on the function of counting in solving number-related reasoning tasks such as in doing arithmetic. The counting tasks used do not require children to relate their counts to adding and subtracting (e.g., Carey, 1998; Fluck \& Henderson, 1996; Frye, Braisby, Lowe, Maroudas, \& Nicholls, 1989; Fuson, Pergament, Lyons, \& Hall, 1985; Wynn, 1990). Debates continue as to whether and when children understand their

${ }^{*}$ Corresponding author. Tel.: +1-732-445-6154; fax: +1-732-445-6715.

E-mail addresses: osnatzur@ucla.edu (O.Zur), rgelman@ruccs.rutgers.edu (R. Gelman). 


\section{Counting and Arithmetic Principles}

- COUNTING PRINCIPLES - HOW TO'S

- 1. One-one

- 2. Stable order

- 3. Cardinal

\section{- PERMISSIONS}

- 4. Order Irrelevance

- 5. Item Kind Irrelevance

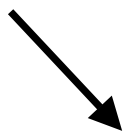

Numerons that stand for the cardinal value $(\mathrm{N})$ of counted collection of discrete entities. If numer are mapped to counting list, cardinal count word re-represents cardinal quantity

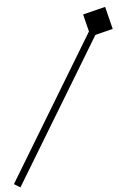

- ARITHMETIC REASONING PRINCIPLES

- ADDITION \& SUBTRACTION

- A/S applied to cardinal values

- If A/S, relevant operation and $\mathrm{N}$ changes, cardinal value $>$ or $<$

- IF $\mathrm{N}$-irrelevant operation, $\mathrm{N}$ is same $(=)$ cardinal value

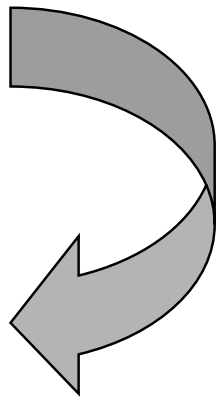

Fig. 1. A schematic representation of the relationship between the Gelman and Gallistel (1978) counting and arithmetic principles.

verbal counts, with many researchers holding that early counts are nothing but rote learning (Fuson, 1988; Sophian, 1998). We submit that it is hard to address these questions without using tasks that relate counting to the goal of doing arithmetic. This is because counting and arithmetic principles are mutually constraining (Fig. 1). Counting must yield cardinal values that can be added, subtracted, or ordered, in accordance with the arithmetic reasoning principles.

Some have studied preschool counting in the context of arithmetic tasks (see Gelman, 1972, 1978; and others in this volume). The number of such investigations is small, especially as compared to the vast number on counting and/or numerical ordering. Current theoretical debates about early competence take place almost entirely in terms of results from one or another counting task. This is like using a series of first stage displays in a number conservation task and never going on to the transformation part of the task. Gelman (1972) concurred with Piaget on a general point: Failure to consider a child's response to transformations renders ambiguous the interpretation of knowledge.

We searched for a new counting task, one that requires young children to relate verbal counting to arithmetic reasoning, by observing a 4-year-old preschool classroom. Our goal was to identify activities in which children have the opportunity to embed their knowledge of counting in a number-relevant reasoning task, as opposed to a rote count routine.

\subsection{Evolving the arithmetic-counting task}

We zeroed in on a classroom number game. In what we dub arithmetic-counting, counting functions to achieve a cardinal value to an addition or subtraction problem. Fig. 2 shows a child participating in 


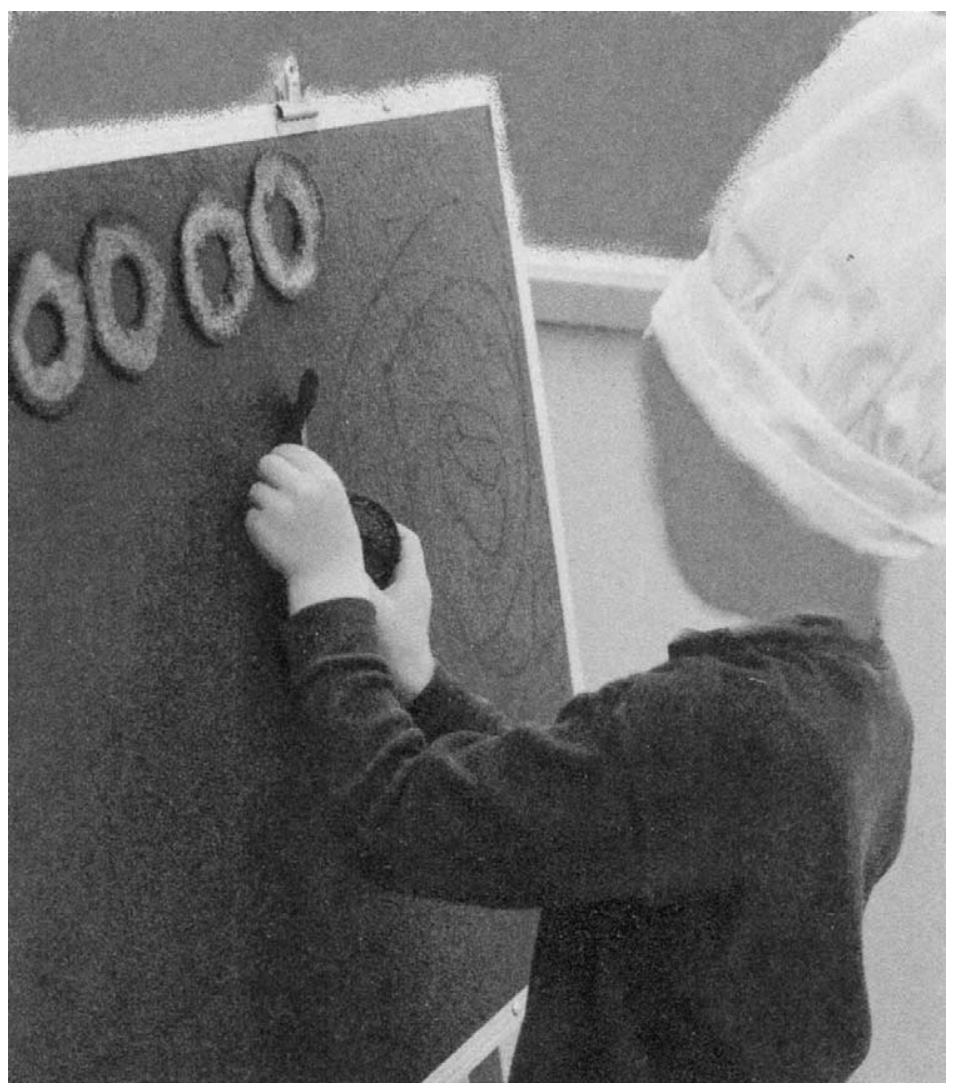

Fig. 2. Preschool child following teacher's in-class request to place $N$ more 'donut' on the board.

an example of the number game the teacher introduced during circle time. She did this while placing the mentioned items, e.g., donuts, on an upright flannel board. The teacher first had the class determine how many objects were in a collection (e.g., that there were 10 donuts). Next, while the class sang the number song (e.g., "Ten little donuts in the bakery shop ... along came John with 2 pennies to pay. He got two donuts and walked away"), a child was asked to remove or add a certain number of items (e.g., 2 donuts). The remaining objects were visible. Then children called out the resulting answers, without having counted. When three donuts were removed, various children said, "Maybe eight"; "Nine"; "Seven"; "No, nine"; "I think eight"; and so on. Another child turned to the teacher and said, "You count!" Instead she had them count and decide who was right.

Since the class quickly caught onto the rules of game we asked the teacher to move to a systematic sequence, adding and subtracting only one each time from a number no larger than 7 . Children were asked first to predict the addition or subtraction result and then to check by counting. We were very impressed with the staying power of these young children and their competence. So we suggested that she alter the problems' difficulty level by gradually increasing the set size (from 7 to 15 and varying the number added or subtracted from the array $(N= \pm 1,2$ or 3$)$. Now, every time the main refrain of the song repeated, children predicted the answer to a problem, and then checked their prediction by counting. Only when children agreed on the answer did they continue to the next refrain. The prediction and checking 


\section{Predict and Check Procedure}

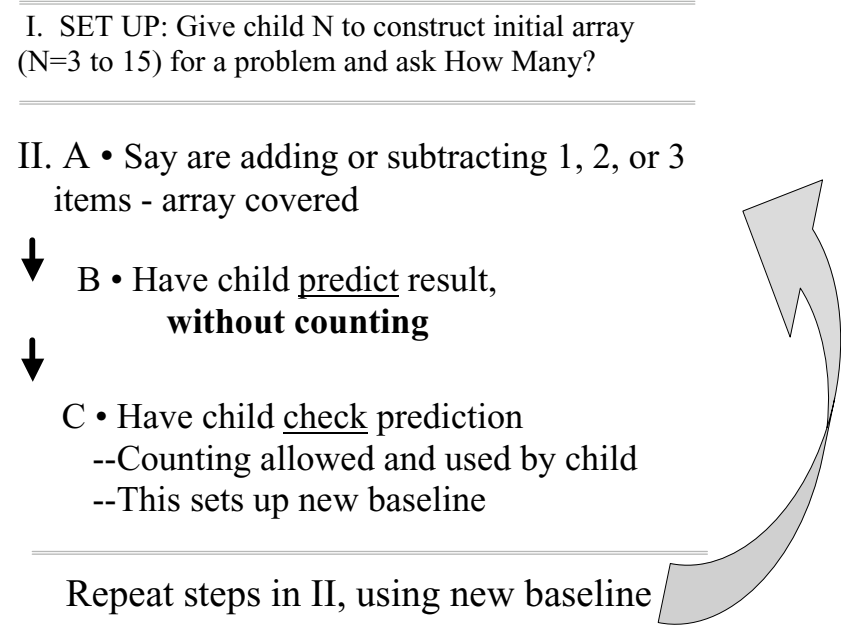

Fig. 3. Schematic outline of the predicting-counting experimental procedure used in all experiments.

components became integral parts of the number game as well as the framework for a new experimental paradigm. Fig. 3 illustrates the resultant structure and sequence of steps within a given problem.

To start a problem sequence, children answer a "How many?" (HM) question and generate an initial starting value by counting the array. This done, addition or subtraction commences. Now children have to monitor whether addition or subtraction is performed, and predict the resulting $N$. Next, they count to check their prediction. The value obtained for a check then serves as the start of the next problem, and the predicting and checking-count procedure repeats.

The group's in-class performance on both addition and subtraction of the arithmetic-counting song was impressive. Children quickly caught onto the distinction between "predicting" and "checking." Their predictions were consistent with the arithmetic principles, that addition increases and subtraction decreases numerosity, even with set sizes as large as 15 . Furthermore, many of the children's predictions and checking counts were accurate or close to accurate.

\subsection{From the classroom to the experiment}

There are several questions one can ask about the in-class activity. First, did individual children share what appeared to be the group competencies? Second, how will children who do not have the in-class experiences perform? Third, what can be expected of even younger children? Three experiments addressed these issues. Experiment 1 (Group-to-Individual) tested the experienced children with a procedure that closely paralleled the classroom set-up. A Follow-up study was done with children in a different class to see if we could replicate our findings with children who did not have in-class experience. Finally, in a third study, 3- to 3.5-year-olds were run in a study with a restricted set size range. They too had no in-class experience.

All studies employed the "Arithmetic-counting" procedure. Our goal was to determine whether children could count with understanding when they do so in the service of solving addition and subtraction 
problems. By using the arithmetic-counting task to achieve this goal, we also can ask whether children can distinguish between "predicting" and "checking." To predict, the child had to generate a cardinal value, to estimate the answer before counting the set. Checking, on the other hand, involved the systematic use of counting as a method for verifying a prediction.

The participants in the first two studies attended a child-care site on a university campus in West Los Angeles and came from an ethnically and racially mixed sample of parents who were students, employees and faculty on the campus. Some children were on scholarship or had their fees subvented from grants for families with economic need. Still, the sample was predominantly middle-class. The 3-year-olds attended a summer day camp in the West Valley of Los Angeles. Signed permission was obtained for all children.

\section{About the research}

Children in the first study (Group-to-Individual) were run in a procedure that stayed as close to the evolved classroom procedure as possible. We needed to determine how many children contributed to the overall impression of successful classroom engagement. Therefore, as in the class, the arrays were not covered during the prediction phase and the experimenter mentioned the use of counting to check a prediction.

The second study (Follow-up), used a more stringent procedure. On any given trial, after items were added or subtracted, the resultant set was covered, and only then children were asked to predict. During the checking phase, children had to decide, on their own, to count to check. The researcher simply said, "Let's find out" or "Let's check your prediction." These changes, in combination with the absence of in-class pre-test experiences, allowed us to obtain evidence about the effects of experience on counting, skill at arithmetic, and the general skills of predicting and checking. A third study with young 3-year-olds asked whether even children this young can count with understanding as evidenced by their ability to distinguish between predicting and checking.

\subsection{The 4-year-old studies}

\subsubsection{Details}

The children in the first and second study ranged in age from 3.5 to 5.5 years $(M=4.9)$ and 3.11 to 5.4 years $(M=4.7)$, respectively. There were six boys and eight girls in the first study and equal numbers of boys and girls (six each) in the second study. Children were individually tested in a two 15-min sessions on two different days in a quiet place at their schools. The sessions were video- and audiorecorded.

A common experimental design was used in both 4-year-old studies. This included three problem-sets administered in an easy-to-hard sequence (Table 1) with Problem-sets 1,2 and 3 run successively. The third set was run on a different day. The maximum set size encountered was 10, 12 or 15 on Problem-sets 1, 2, and 3, respectively. Problem-set 1 (subtract-easy) and Problem-set 2 (subtract-hard) had only subtraction problems. Problem-set 3 [Mixed A/S, $N \pm(1,2,3,4,5)]$ included addition and subtraction items and was based on Eric Carl's (1972) The Rooster Who Set Off to See the World, a story that the teacher read several times to her class. There were two versions of the story (familiar and novel order), with the first following the original story in the book as well as classroom activities paired with it. The second version 
Table 1

Problem sets and results for both 4-year predict and check experiment

\begin{tabular}{|c|c|c|c|c|c|c|}
\hline \multirow[t]{2}{*}{ Problem-sets } & \multicolumn{3}{|c|}{ Experiment 1 Group-to-Individual } & \multicolumn{3}{|c|}{ Experiment 2 Follow-up } \\
\hline & Mean prediction & S.D. & Correct $(\%)$ & Mean prediction & S.D. & Correct $(\%)$ \\
\hline \multicolumn{7}{|c|}{ Problem-set 1: Subtract Easy $(N-1)$} \\
\hline $10-1$ & 8.3 & 1.0 & 0.66 & 7.4 & 2.7 & 0.67 \\
\hline $9-1$ & 7.5 & 1.0 & 0.77 & 7.9 & 0.8 & 0.67 \\
\hline $8-1$ & 7.2 & 1.3 & 0.77 & 6.7 & 0.6 & 0.82 \\
\hline $7-1$ & 5.8 & 0.4 & 0.84 & 6.3 & 0.9 & 0.83 \\
\hline $6-1$ & 5.2 & 1.0 & 0.84 & 5.7 & 2.7 & 0.58 \\
\hline $5-1$ & 4.2 & 0.9 & 0.84 & 4.2 & 0.6 & 0.92 \\
\hline \multicolumn{7}{|c|}{ Problem-set 2: Subtract Hard $(N-1,2,3)$} \\
\hline $12-1$ & 10.0 & 4.3 & 0.22 & 10.6 & 4.4 & 0.20 \\
\hline $12-3$ & 7.8 & 2.2 & 0.40 & 7.8 & 1.3 & 0.40 \\
\hline $11-2$ & 8.4 & 1.7 & 0.33 & 8.2 & 2.0 & 0.00 \\
\hline $9-3$ & 7.2 & 1.5 & 0.22 & 7.7 & 3.1 & 0.50 \\
\hline $9-2$ & 8.0 & 0.7 & 0.40 & 7.2 & 0.8 & 0.50 \\
\hline $7-1$ & 7.4 & 1.9 & 0.60 & 5.5 & 1.2 & 0.83 \\
\hline $6-2$ & 3.7 & 0.5 & 0.71 & 3.9 & 0.3 & 0.92 \\
\hline $4-3$ & 1.0 & 0.0 & 1.00 & 1.0 & 0.0 & 0.00 \\
\hline \multicolumn{7}{|c|}{ Problem-set 3: Mixed A/S $(N \pm 1,2,3,4,5)$} \\
\hline \multicolumn{7}{|c|}{ Familiar Version } \\
\hline $1+2$ & 3.0 & 0.0 & 1.00 & 3.0 & 0.00 & 1.00 \\
\hline $3+3$ & 5.6 & 1.6 & 0.38 & 5.7 & 0.50 & 0.71 \\
\hline $6+4$ & 8.8 & 1.4 & 0.50 & 9.4 & 1.6 & 0.43 \\
\hline $10+5$ & 12.6 & 3.3 & 0.13 & 13.5 & 1.9 & 0.43 \\
\hline $15-5$ & 10.5 & 2.3 & 0.75 & 10.3 & 1.5 & 0.71 \\
\hline $10-4$ & 5.8 & 1.3 & 0.62 & 6.0 & 0.8 & 0.71 \\
\hline $6-3$ & 3.0 & 0.0 & 1.00 & 3.0 & 0.0 & 1.00 \\
\hline $3-2$ & 1.0 & 0.0 & 1.00 & 1.0 & 0.0 & 1.00 \\
\hline \multicolumn{7}{|c|}{ Novel Version } \\
\hline $1+5$ & 7.2 & 1.6 & 0.50 & 7.5 & 1.9 & 0.50 \\
\hline $6+2$ & 8.8 & 1.3 & 0.60 & 7.8 & 0.4 & 0.80 \\
\hline $8+4$ & 11.5 & 1.5 & 0.33 & 12.0 & 3.8 & 0.00 \\
\hline $12+3$ & 13.5 & 2.0 & 0.50 & 12.8 & 2.7 & 0.00 \\
\hline $15-2$ & 12.1 & 1.6 & 0.00 & 13.0 & 3.3 & 0.00 \\
\hline $13-3$ & 9.3 & 1.8 & 0.50 & 9.2 & 3.7 & 0.00 \\
\hline $10-5$ & 5.1 & 0.4 & 0.83 & 6.2 & 2.7 & 0.40 \\
\hline $5-4$ & 1.0 & 0.0 & 1.00 & 1.0 & 0.0 & 1.00 \\
\hline
\end{tabular}

altered the animal combinations and order of addition and subtraction problems to create a novel problem set. ${ }^{1}$ Three children recruited for the second study were dropped due to illness or response bias.

The novel problem set allowed us to assess whether transfer occurred. The problems in each of the problem-sets are listed in Table 1.

\footnotetext{
${ }^{1}$ For version II, the original order in which the kinds of animals were added or subtracted from the array was changed to generate novel combinations of animal kinds and set sizes. Children were randomly assigned to either version I or II.
} 


\subsubsection{Procedure}

The flannel board and felt pieces were the same ones the teacher used in class when presenting number songs/games. In addition, a farmer's hat and a caterpillar puppet were used on Problem-set 1, and 12 pennies and a baker's hat were used on Problem-set 2. Felt pieces were placed on flannel board, as they were in the teacher number songs/games. These pieces included 10 green apples $(7 \mathrm{~cm} \times 7 \mathrm{~cm})$, a tree, and 10 yellow ducks $(5 \mathrm{~cm} \times 5 \mathrm{~cm})$ on Problem-set 1,12 brown donuts $(9 \mathrm{~cm})$ on Problem-set 2 , and 1 rooster, 2 cats, 3 frogs, 4 turtles, and 5 fish (each about $7 \times 9 \mathrm{~cm}$ ) on Problem-set 3 . Animals of the same kind were identical. In addition, a farmer's hat and a caterpillar puppet were used on Problem-set 1, and 12 U.S. pennies and a baker's hat were used on Problem-set 2.

A test cycle started with the opening request of "How many?" to establish the numerosity of the initial array, and the child was encouraged to count to obtain the answer. This was the only time in a problem-set that the How Many? question was featured. The task continued with a recursive sequence of arithmetic trials. Each trial constituted the re-current cycle of a transformation, prediction, and checking phase (Fig. 3). For the transformation, items were added to or subtracted from the array (depending on the task kind) by the experimenter. The child predicted the number of items in the transformed array, and only then checked his/her prediction by counting the items. The starting array on the next trial within the problem-set was always that same as the post-transformed array that resulted as a consequence of the previous arithmetic operator. The problems in each problem-set were embedded in a story.

On each problem-set, the child was treated as an active agent in the scenario (e.g., "Let's pretend you are a baker"). (The story used in each of the three problem-sets is available from the authors.) If a child tried to count the array before making a prediction, the experimenter said, "Wait, before you count, let's make a prediction first. How many apples/ducks are left?" and only then did she let the child count the array to check his/her answer. When the occasional child did not make a prediction, E said, "Any prediction is good."

\subsection{Four-year-old results}

\subsubsection{Counting for the "How Many?" set-up and checking phases}

The assessments of counting came from the How Many? (HM) question at the beginning of a problem-set sequence, as well as from the checking phase. The initial sets had 10 (Problem-set 1) and 12 (Problem-set 2) felt items. The child first placed the items on the board, and then was asked the opening question, for example, "How many apples do you have on your tree?" All children in the Group-to-Individual study and all but one child in the Follow up study successfully counted up to 10 or 12 .

We were especially interested in whether the children would restate the last counting tag in their list after counting to answer the initial How Many? Question. Many who study early math require that a child repeat their last count word, e.g., "one, two, three—three," when asked "How Many?"(HM). The claim is the child lacks a cardinal principle if she does not end by announcing the cardinal value, by repeating their last count word. When preschool children are asked the HM question, they seldom restate the last counting tag (e.g., Fluck \& Henderson, 1996; Fuson, 1988; Wynn, 1990). Our results concur. None of the children in the first study, and only one Follow up child repeated the cardinal value (10) after counting out loud up to 10 in Problem-set 1. On Problem-set 2, however, some of the Group-to-Individual children did repeat the cardinal value after the initial count. Four children restated the cardinal value (12) and three others said "a dozen" after counting. (The term "dozen" had been introduced to the class a few months earlier.) None of the children in the Follow-up study restated the cardinal value (12) in Problem-set 2. 
During the checking phase of a trial, all children in the Group-to-Individual study and in the Follow-up study counted to check. The fact that all children in the Follow-up study spontaneously counted to check their predictions is particularly impressive given that in this study the experimenter did not suggest this strategy. Children counted correctly on the majority of checking counts. On the Group-to-Individual study, children miscounted on $4 \%$ of the checking trials but were correct on all but $2(N=15)$ trials when they recounted more slowly. (The errors were mainly on set-sizes $=$ or, $>9$ ). Similarly, the majority of the Follow-up children counted without error; those who erred did so on set sizes of 10 or more. When the experimenter hinted that they could recount slowly, they did so without error. Again, the tendency to restate the cardinal number after a count was rare-it only happened in the Follow-up study and then on only one trial. However, as we shall see, the standard interpretation of such HM task response patterns, is probably wrong.

\subsubsection{Predictions}

Review of the videotapes revealed that children followed instructions and treated predictions differently than checks. When making a prediction, children typically replied immediately with a cardinal value. This contrasts with the fact that they always counted during the checking phase of the trial. This pattern of results held for children in the Follow-up study, even though they did not have prior experience with the task procedure. These children made cardinal value predictions on all but $2 \%$ of the problems (four trials).

Prediction answers were scored at three different levels of stringency: (1) the directionality, that is, whether children's predictions were in the arithmetically correct direction for either an addition or subtraction problem; (2) whether the answer $=N \pm 1$; and (3) perfect. The overall results, across all problem types, for each level of strictness are shown in Fig. 4.

As is evident in the left most bars of Fig. 4 children's predictions in both experiments were very likely to be in the right direction for each problem-set. Thus, the children knew that addition and subtraction increases and decreases the value of $N$ and were able to give reasonable answers. We note that the latter result reveals considerable knowledge about the relative ordering value of different count words-even when set sizes go beyond the small number range, especially since it was obtained in both studies.

As the middle bars of both the top and bottom halves of Fig. 4 show, for each problem-set, when the accuracy criterion is $N= \pm 1$, success levels are lower than when the criterion is Correct Direction. They also are better than those requiring perfect answers. Over $70 \%$ of the children's predictions in both of the studies deviated from the correct answer by only \pm 1 . In the first experiment, the mean average distances from the correct answers were 0.31, 1.04, and 0.95, respectively, and the percent correct predictions were 78, 54 and 60\%, for Problem-sets 1,2, and 3, respectively. The comparable scores for those in the Follow-up study were $0.60,0.86$, and 1.06. The related percent correct predictions were 70, 64 and 53\%, for Problem-sets 1, 2, and 3, respectively. Children's performance on each problem in the three sets is presented in Table 1.

\subsubsection{The effect of experience on prediction abilities}

As shown in Fig. 4, the overall pattern of scores for both groups is very similar. Still, there were differences in performance between the two experiments. First, as expected, there was a difference in performance on the $(N-1)$ problems in Problem-set 1 . The majority of counting errors of the Follow-up group (10 out of 13) were on the large set size problems, with a set-size larger than 10 . The children in the second study had no prior experience with these problems and first encountered them on the $N-1$ set. Although the predictions for the Follow-up experiment were in the right direction, the answers were not as 

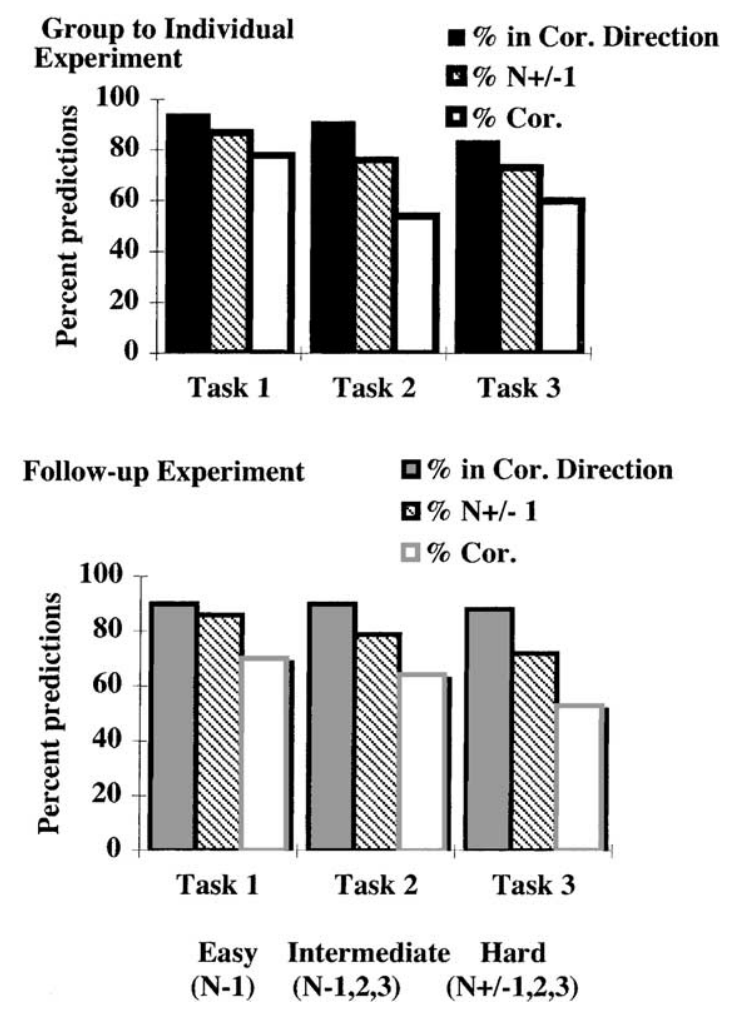

Fig. 4. Percent children in both 4-year-old experiments who met one of three criteria: their predictions were in the correction direction (left most bars); within one of the correct answer (middle bars); or correct (right-hand bars).

close to the correct answers given by the children in the first study. The average absolute deviations from the correct answer were 0.31 and 0.60 for Experiments 1 and 2, respectively), $t_{(24)}=1.95, P<0.05$.

A second difference showed up between the two studies on Problem-set $3(N \pm 1,2$ or 3$)$ - Novel Version. This problem-set was novel for the children in both experiments, particularly for problems where the initial set and/or answer was larger than $8(8+4,12+3,15-2$ and $13-3)$. Yet, the children who had related in-class experience were more likely than children in Follow-up study to make correct predictions. Predictions on these problems by the Follow-up children were in the right direction, but none were accurate, as can be seen in Table 1. These children were also less likely than those in the initial study to make predictions close to the correct answer, $t_{(9)}=2.9, P<0.01$. The average distances from the correct answer on these problems were 1.3 and 2.6 for the first and second studies, respectively.

\subsubsection{Solution-paths}

To find out how the children arrived at their answers, prediction behaviors and strategies were reliably coded from audio and/or videotapes of the sessions. Some of the children predicted quickly; others took time. Individual prediction times were coded as either fast or delayed. Fast predictions were given within $2 \mathrm{~s}$ or less after the request for a prediction. The number of fast answers decreased across successively more difficult problem-sets. The respective values for fast predictions in the first study were 60, 54 and $45 \%$ of the trials in Problem-sets 1, 2, and 3. Those for the Follow-up study were 60, 55 and 30\%. The 
fast answers were more frequent on small set size problems on all three sets. In contrast, the delayed answers were more frequent when children solved large set size problems. On Problem-set $1(N-1)$, for example, the percentage of subjects who answered fast decreased from 71 to $21 \%$ to as the problems' set-size increased.

To our surprise, there was a relation between the time to respond and the accuracy level of the predictions. Fast predictions were more likely to be correct than were delayed predictions, $\chi_{(1)}^{2}=29.9, P<0.01$. This was true for every child and every problem-set. Such results parallel findings reported by Siegler and Jenkins (1989) for older children. Those who reply fast are assumed to use a mental addition or subtraction strategy. Overall, $81 \%$ of the fast answers for children in the Group-to-Individual study were correct. In contrast, only $48 \%$ of the delayed answers provided by these same children were correct. The comparable figures for predictions on each task were 93, 70 and $80 \%$, for fast responses, and 65, 35, and $43 \%$, for delayed responses, on Problem-sets 1,2, and 3, respectively. If these children gave a fast answer, they were likely to be correct on both large and small set size problems. There were 125 large set-size trials. Children gave delayed answers on 77 trials and fast answers on 48 trials. Only 39\% of the delayed answers were correct, whereas $66 \%$ of the fast predictions were correct.

A similar pattern of results was observed in the Follow-up experiment. Overall, $86 \%$ of children's fast answers were correct. In contrast, only $46 \%$ of the predictions that were made following a delay were correct. Fast predictions were significantly more likely to be correct, $\chi_{(1)}^{2}=37.3, P<0.001$. As in Experiment 1, it held true on small and large set-size problems. On the large set-size problems, $65 \%$ of the fast answers were correct, but only $31 \%$ of the delayed predictions were correct.

We observed four strategies for delayed predictions: (i) Tried to look: In the first study, although the child did not count, she tried to peek at the uncovered items before giving an answer. Such efforts led the experimenter to say, "Wait, don't look." During the first experiment, this happened on $21 \%$ of the trials of each of the problem-sets, and was more frequent on small set-size problems (e.g., $1+2,4-3$ ). The percentage of subjects who did this increased from $21 \%$ on Problem-set 1 to $64 \%$ on Problem-set 3 ; (ii) Wanting to count: The child asked if she could count the array and was told no. This happened on a minority of the trials in the first study, 29, 23, and $11 \%$ for Problem-sets 1, 2 and 3. It was most frequent on the first and second trials of the $N-1$ subtraction sets for which the largest set size problems were presented first (e.g., $12-1,11-2$ ). On Problem-set 3 wanting to count was more likely on problems with the larger addend, or subtrahend (e.g., $3+3,6+4,1+5,10-4)$. Wanting to count behavior was less frequent during the Follow-up study, occurring on $14 \%$ of trials. Again, prediction trials that involved delays were most likely to induce the "wanting to count" behaviors. It was most common on the initial prediction trials of each task; (iii) Counting aloud: Two subjects counted aloud to figure out the answers. One of them counted backwards (on subtraction problems), and the other used a "count all" (Fuson, 1988) solution; and (iv) Fingers: When this strategy was used, a child did not count verbally, but used her fingers to either show or figure out the answer to the problem. Even though we did not see this in their class, 6 of the 14 children in the first study used their fingers on at least one prediction trial. One of these children used his fingers on all trials. None of the Follow-up subjects adopted a finger strategy.

\subsection{Summary of the 4-year-old experiments}

The pattern of results for both experiments is comparable. Differences are localized in ways that reflect the in-class experience of children in the first study. These children had some (albeit not a great deal) 
experience with the problem formats and novel large set-size problems. Their predicting and counting scores were more accurate than the children who did not share this prior experience. Nevertheless, the novices did rather well. It is particularly relevant that these children, who had no previous experience with the problems, made many fast predictions, with the majority of these quick responses being correct, even for large set-sizes. Thus, the ability to give fast responses for arithmetic problems cannot simply be a matter of in-class practice with the problem form. In addition, these children knew to count in order to check their predictions - even though they were not told to do so. But what should we expect for still younger children? Are they able to relate cardinal values to addition and subtraction? If so, do they also use different strategies for predicting and checking?

\section{The 3-year-old experiment}

In studies of counting, young children are likely to fail the "How Many" and Give X (to a puppet or experimenter) tasks (Fluck \& Henderson, 1996; Wynn, 1990). Such evidence is used to conclude that there is lack of initial understanding about verbal counting and enumeration. However, at that same young age, they can solve addition and subtraction problems with small numbers (e.g., Gelman, 1972; Hughes, 1981; Starkey \& Gelman, 1982). In the latter studies, children were presented with a transformation (either addition or subtraction), and had to indicate the numerosity of the resultant array, either verbally by stating the numerosity of the transformed array, or non-verbally via surprise reactions or the creation of an array with the correct number of items. Our arithmetic-counting task incorporates both verbal counting and verbal arithmetic. If young 3-year-olds can succeed on it, we will have further evidence that they count with understanding, that is, relate cardinal values to addition and subtraction.

\subsection{Details}

Eighteen children were recruited from a preschool summer camp in the West Valley area of Los Angeles. Fifteen (eight boys, seven girls), aged 3.1-3.7 ( $M=3.4$, median = 3.5) completed the experiment. Their ages were evenly distributed across the sample range with 10 falling between the ages of 3.1 and 3.5. Given the target age range, we chose to restrict our set-size range and used problems with a set-size up to $N=5$. Given our interest in whether the 3-year-olds could generate cardinal values to make a prediction, and only then count to check their predictions, the array always was covered during the transformation and prediction phases.

\subsubsection{Pre-test session}

The experimenter joined the children's morning camp activities for about four days. On a separate day there was an individual warm-up session that provided some experience with predicting and counting. During this session children had a chance to either "count" or "predict and count" the number of parts on familiar (e.g., hand, car) and unfamiliar objects (e.g., an apple, half egg-carton. For example, in the "count" condition the experimenter presented the object "Here is a car. How many wheels does the car have?" In the "predict and count" condition-the experimenter presented the child with the object, then the object was covered and the experimenter said "How many wheels does the car have? Can you make a prediction?" After the child predicted, the object was uncovered and the child was asked to count and find out. The order of these tasks was counterbalanced. We intentionally chose items such that a child 


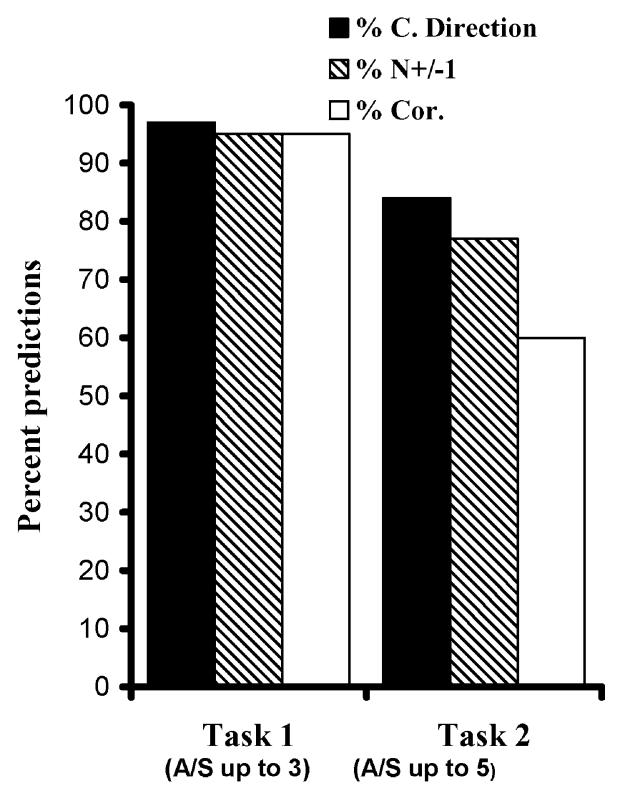

Fig. 5. The young-three-olds' predictions for $N+1$ and $N-1$ problems.

was likely to make a correct prediction for some but not for others. Our goal was to show the child that a prediction could either be correct or not.

\subsection{Experimental session}

All children were given all problem-sets, in an easy-to-hard sequence. Problem-set 1 (Addition/ Subtraction up to 3) was presented first, and was followed immediately by Problem-set 2 (A/S up to $5)$. Both problem-sets included addition and subtraction problems embedded in songs, and used the counting-arithmetic sequence schematized in Fig. 3. The materials used for the experiment included two sets of small plastic animals, three green frogs on Problem-set 1 (A/S up to 3), and six yellow ducks on Problem-set 2 (A/S up to 5). Each animal was about $5 \mathrm{~cm}$ long, except for one duck that was slightly bigger than the rest and served as "mother duck." A piece of white cloth was used to cover the array. Fig. 5 shows the problems in the context of the results. The testing sequence followed that used in the Follow-up study with older children, save for the change in items. Counting was correct on all trials, which made it easy for the experimenter to positively reinforce a child. When a child's prediction for a trial was not correct, the experimenter finished the counting part of the trial by saying "So, now we have" or "What did you just find out?"

\subsection{Results}

\subsubsection{Pre-test predicting and counting}

In making pre-test predictions, children usually generated a cardinal value (e.g., "two," "four"); but on a small minority of their trials they counted aloud up to the value (e.g., "one, two, three," when predicting there would be 3 , say seeds in an apple). Some children always gave a count-prediction, some 
children consistently generated a cardinal value and still others used both solutions. The ranges and median prediction for each object type were not random or arbitrary. Predictions about the number of parts of more familiar objects (e.g., airplane, car, hand) were very close to the correct value and the range was relatively small. In contrast, when children predicted the number of parts for less familiar items (e.g., egg carton), or for items where it was impossible to know the exact value (e.g., number of seeds in an apple), the prediction range was reliably bigger. In addition, when children were presented with a less familiar object, they were more likely to point to the covered object and tell the experimenter that they wanted to count its parts.

Children who had some prediction experience before they counted were correct on $79 \%$ of their initial efforts to count a display. If we include recounts after the experimenter provided hints (a request to count more slowly, pointing to the parts while children counted, or a re-statement of the first count word), the accuracy level is $89 \%$. Of interest is the fact that those who only had pre-test counting but no prediction experience did not count as well. Overall, 59\% of their starting counts were correct. If hints are allowed, the accuracy level is $71 \%$. For both groups, the majority of the counting errors occurred when children counted the seeds in the apple, or the objects with six parts (egg carton and truck). It usually involved counting one less or one too many.

\subsubsection{Prediction phase}

All of the children appeared to understand what "making a prediction" meant whether or not they had predicting experience in the warm-up phase. Children made predictions on all but one of the arithmetic trials. On $87 \%$ of the arithmetic trials, children were able to alternate between generating a cardinal value during the prediction phase, and "counting" on their own during the checking phase. Ten of the 15 children always stated a cardinal value during the prediction phase; the remaining five children generated a cardinal value on at least $60 \%$ of the arithmetic trials. On only $13 \%$ of the trials did children fail to offer a cardinal value as a prediction. On these trials, they either counted out loud up to the predicted value (10\%), or held up $N$ fingers (3\%). On $24 \%$ of the arithmetic trials, children both stated a cardinal value, and held up $N$ fingers.

Fig. 5 shows that about $90 \%$ of children's predictions were in the right direction, although they were more likely to be in the right direction on Problem-set 1 than on the Problem-set 2 with the larger values. Like the 4-year-olds, these young children showed an implicit understanding of the principled requirement that addition increases numerosity and subtraction decreases numerosity. The figure also shows that a large proportion of the predictions were only off by \pm 1 . Still, as the set-size increased (Problem-set 2: A/S up to 5), the proportion of predictions that was accurate or off by one decreased. As in Experiment 1 , children's predictions increased or decreased with the value of the answers to the problems, and the predicted values were close to the problems' answers. After a child made a prediction, the experimenter uncovered the array and asked the child to "count and find out." On 92\% of trials, children started out by counting the array. On the remaining trials, children looked at the items, stated the cardinal value, and only then counted the array. Children were extremely good at counting the array. Counting was correct on all trials but one. As in Experiments 1 and 2, children rarely spontaneously repeated the cardinal value after counting the array ( $7 \%$ of trials); only one child responded with a cardinal value after counting. On $20 \%$ of trials, the experimenter asked the child "How many?" after the checking-count. In this case, the children responded with the correct cardinal value on all but two trials.

As in the two experiments with 4-year-olds, predictions were either fast or delayed, and the number of fast predictions decreased from Problem-set 1 (A/S up to 3) to Problem-set 2 (A/S up to 5), as the task difficulty level increased. These young 3-year-old children gave fast answers on 78 and $51 \%$ of 
Problem-sets 1 and 2, respectively. These fast answers were given either just after the song ended, or after the experimenter re-stated the problem at the end of the song.

Once again we found a relation between time to respond and accuracy level of the predictions. Fast predictions were more likely to be correct than delayed predictions. The percentages of correct predictions for fast answers were greater than those given after a delay. This is particularly evident on Problem-set 2 (A/S up to 5); children were correct on $75 \%$ of the fast answers, but only on $45 \%$ of the delayed (short or long) ones. On Problem-set 1 (A/S up to 3), all the fast predictions and those given after a short delay were correct, but only $66 \%$ of the answers after a long delay were correct. Again, when a prediction trial involved a delay, some children tried to count the array (10\% of trials). They usually tried to uncover the array in order to find out the answer. Of course, such efforts were interrupted. The slow-delayed distinction reflects the differential use of a rapid addition-subtraction as opposed to a count-everything strategy in older children. Of interest, is the possibility that same is so for preschool children. This is an unexpected outcome but one that deserves both research and pedagogical attention.

\subsection{Summary}

The foregoing results tell us that the arithmetic-counting task can be used with young 3-year-olds. Even these young children demonstrated an understanding of the distinction between predicting and counting in order to check a prediction. During the prediction phase of each trial, children did not simply count to themselves. All children were able to offer a cardinal value that resulted from an addition or subtraction. Some made comments (hedges) unique to the prediction phase; e.g., "I think four," "Maybe two." The predictions they generated honored the principles that addition increases numerosity and subtraction decreases numerosity. They also made reasonable predictions - ones which were correct or in the correct direction. Even though the experiment involved small set-sizes, it is unlikely that these young children solved the problems by using a perceptual, non-numerical procedure, often called "subitizing." After all, the arrays were covered. Further, like the older children (on larger set-sizes), they exhibited variability in the time they took to answer. When the children made a delayed answer, they attempted to apply verbal counting strategies; they either asked the experimenter if they could count, or wanted to uncover the array in order to find out the answer. Children's attempts to count indicate an understanding of the function of counting in solving an arithmetic problem. During the checking phase, children did not simply repeat their prediction; they counted the arrays, and their counts were usually correct. Further, the answer to a count won out.

The arithmetic-counting task uncovered evidence that the young 3-year-olds in our study used counting in a numerical reasoning task. It brought out young children's ability to use count words in an arithmetic context, where counting is in the service of solving addition and subtraction and renders an arithmetically meaningful outcome. In this regard, the "arithmetic-counting task shared a key characteristic with Gelman's "magic-task" (Gelman, 1972, 1993). On both tasks, children were required to relate their implicit knowledge of counting to arithmetic principles. Straightforward counting tasks (e.g., HM) or other arithmetic tasks used with young children do not share this feature.

\section{Theoretical implications}

We combined a naturalistic study in the preschool classroom with an experimental method in order to ask whether 3- and 4-year-old children can count with understanding. The in-class observations led to a 
partnership with a terrific teacher who went along with our somewhat unusual requests as to how to alter the arithmetic-counting task. We all learned that the children could do more than expected. A similar pattern of results was obtained with children who had no relevant in-class experience. All groups of children, even the those in the young 3-year-olds study could generate arithmetically reasonable predictions, that is ones that were in the right direction. Importantly, many gave predictions that either were correct or very close to the correct answer and then chose to rely on their correct counting checks for the answer.

The number-game we were lucky to witness in a classroom setting, and then expand, was assimilated with relative ease. It did not require any formal instruction and therefore meets a commonly assumed criterion regarding the choice of a preschool 'curriculum'. A short pre-test experience sufficed even for young 3-year-olds. Children were engaged and motivated to take turns and solve the number-problems. What is it about the arithmetic-counting task that brings to the fore their competence? Number-games of this kind allow for the use of an implicit or intuitive sense of numerical operations. In solving an arithmetic problem, children use counting to satisfy a meaningful goal — to find the number of objects in the transformed set. In this process, a higher-level conceptual knowledge of addition and subtraction guides and constrains children's counts. As suggested by Gelman (Gelman, 2000; Gelman \& Williams, 1998), learning in the domain of number benefits from skeletal mental structures that expedite the assimilation and use of domain-relevant knowledge. The arithmetic-counting games provide input that map onto existing implicit number knowledge structures. Our position is consistent with Siegler and Jenkins' (1989) view that the process of arithmetic-problem solving is constrained by a goal sketch and Saxe's (1991) emphasis on social goals for arithmetic. Counting tasks that are not embedded in number reasoning contexts do not share these features. This is why we are skeptical about conclusions based only on counting or comparison tasks.

We end with a word of caution: Many investigators hold that early counting lacks any appreciation of the quantitative function of counting (e.g., Baroody \& Price, 1983; Fuson, 1988; Simon, 1997; Sophian, 1998). We join with Ginsburg (1989) in contesting this position. Nevertheless, we do not mean to say that young children do not have to work at learning the count list. Unfortunately, the count word string is a long serial list that must be mastered. Whenever humans, be they young children or adults, have to learn a serial list there is no substitute for practice. So, we recognize that practice is necessary. The issue is how should practice be embedded in the preschool day?

Our view regarding the role of practice is this: Since the ultimate goal is to achieve learning with understanding, it is important for teachers to keep in mind the well-known psychological fat that practice is likely to have more effect if it builds upon and extends beginning verbal understandings of the meaning and role of count words. Since the meaning is tied to arithmetic operations, teachers are well advised to relate counting practice to the relationship between count words and the operations of addition, subtraction and ordering.

\section{Practical implications}

The work covered here has direct implications for early number and science curricula. The ease with which young children learn to predict and check can serve teachers in all kinds of ways. For example, in exploring the inside and outside of fruits and vegetables, children made predictions about "what's inside" (Gelman \& Brenneman, in press). We are sure that teachers will outdo us in thinking of ways to use this 
informal learning tool. After all, the success of the work reported depended on our privilege to be able to cycle from the classroom to lab, to classroom, and so on.

The similarities and differences in the pattern of results presented here and in other publications have implications for preschool math programs. First, there is good reason to provide young children with opportunities to work with largish set sizes. Doing so expands the range of arithmetic problems they can handle and encourages the mapping of the counting principles to words that represent larger values. Second, since children are able to relate their counts to arithmetic principle, teachers are well advised to use various counting-arithmetic games. Not only do children catch on, they enjoy them and get meaningful practice at learning the count sequence, their ordering and cardinal meanings and how these relate to addition and subtraction.

\section{Acknowledgements}

Support for this work came from NSF Grants No. SBR-9209741, and FDP-NSF No. SPR9720410 to Rochel Gelman, and a pre-doctoral NIMH training Grant No. USPHS-MH19926 to Osnat Zur. We especially thank Alyssa Lafosse and Andrew Lac, for their help in coding and entering the data; Sara Cordes for thoughtful comments about the manuscript; and Susan Wood, the staff, children, and parents of the Bellagio site of the UCLA Child Care Services, and the Temple Aliyah Nursery School.

\section{References}

Baroody, A. J., \& Price, J. (1983). The development of the number-word sequence in the counting of three-year-olds. Journal for Research in Mathematics Education, 14(5), 361-368.

Carey, S. (1998). Knowledge of number: Its evolution and ontogenesis. Science, 242, 641-642.

Fluck, M., \& Henderson, L. (1996). Counting and cardinality in English pupils. British Journal of Educational Psychology, 66, 501-517.

Frye, D., Braisby, N., Lowe, J., Maroudas, C., \& Nicholls, J. (1989). Young children's understanding of counting and cardinality. Child Development, 60, 1158-1171.

Fuson, K. C. (1988). Children's counting and concepts of number. New York: Springer-Verlag.

Fuson, K., Pergament, G., Lyons, B., \& Hall, J. (1985). Children's conformity to the cardinality rule as a function of set size and counting accuracy. Child Development, 56, 1429-1436.

Gelman, R. (1972). Logical capacity of very young children: Number invariance rules. Child Development, 43, 75-90.

Gelman, R. (1993). A rational-constructivist account of early learning about numbers and objects. The Psychology of Learning and Motivation, 30, 61-96.

Gelman, R. (2000). The epigenesis of mathematical thinking. Journal of Applied Developmental Psychology, 21(1), 2737.

Gelman, R., \& Brenneman, K. (in press). Science learning pathways for young children. Early Child Research Quarterly.

Gelman, R., \& Gallistel, C. R. (1978). The child's understanding of number. Cambridge, MA: Harvard University Press.

Gelman, R., \& Williams E. M. (1998). Enabling constraints for cognitive development and learning: Domain specificity and epigenesis. In D. Kuhn \& R. S. Siegler (Eds.), Cognition, perception and language (Vol. 2, pp. 575-630). New York: Wiley.

Ginsburg, H. P. (1989). Children's arithmetic (2nd ed.). Austin, TX: Pro-Ed.

Hughes, M. (1981). Can preschool children add and subtract? Educational Psychology, 1, 207-219.

Saxe, G. B. (1991). Culture and cognitive development. Hillsdale, NJ: Lawrence Erlbaum Associates.

Siegler, R. S., \& Jenkins, E. (1989). How children discover new strategies. Hillsdale, NJ: Erlbaum.

Simon, T. J. (1997). Reconceptualizing the origins of number knowledge: A "non-numerical" account. Cognitive Development, $12,349-372$. 
Sophian, C. (1998). A developmental perspective on children's counting. In C. Donlan (Ed.), The development of mathematical skills (pp. 27-46). Psychology Press.

Starkey, P., \& Gelman, R. (1982). The development of addition and subtraction abilities prior to formal schooling in arithmetic.

In T. P. Carpenter, J. M. Moser, \& T. A. Romberg (Eds.), Addition and subtraction: A cognitive perspective (pp. 99-116). Hillsdale, NJ: Erlbaum.

Wynn, K. (1990). Children's understanding of counting. Cognition, 36, 155-193. 